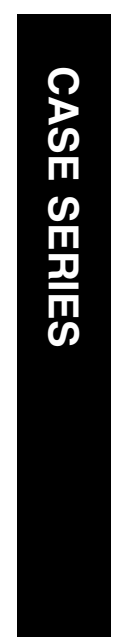

\title{
Cyclodiode laser in the treatment of acute angle closure
}

\begin{abstract}
Aim To describe the outcome of using diode laser transscleral cyclophotocoagulation (cyclodiode laser) as a safe technique in managing acute angle closure refractory to conventional treatment.

Methods This is a retrospective case series from two ophthalmic units in the United Kingdom. Five patients with acute angle closure refractory to medical and laser treatment underwent cyclodiode laser treatment. Demographic information, symptoms, medical and surgical treatment, visual outcomes, and intraocular pressure (IOP) control were recorded.

Case reports All five patients had symptomatic acute angle closure. Conventional

management, including topical and systemic medical treatment, laser iridotomy and laser iridoplasty, did not achieve adequate IOP control or relieve symptoms. Emergency cyclodiode laser treatment was performed within 2-23 days of presentation. All patients subsequently required lensectomy at a later date. At final follow-up (6-14 months), all patients had visual acuity of $6 / 12$ or better with well-controlled IOPs ( $\leq 17 \mathrm{~mm} \mathrm{Hg}$ ). Only one patient was on topical treatment. One patient developed a persistent low-grade anterior uveitis. Discussion Cyclodiode laser is a safe alternative to emergency lensectomy or trabeculectomy in cases of acute angle closure, which do not respond to treatment.
\end{abstract}

${ }^{1}$ Birmingham Midland Eye Centre, Sandwell and West Birmingham Hospitals NHS Trust, Birmingham, UK

${ }^{2}$ NIHR Biomedical Research Centre for Ophthalmology, UCL Institute of Ophthalmology \& Moorfields Eye Hospital, London, UK

${ }^{3}$ Moorfields Eye Hospital NHS Foundation Trust London, UK

Correspondence: A Manna, Birmingham Midland Eye Centre, Sandwell and West Birmingham Hospitals NHS Trust, Dudley Road, Birmingham, West Midlands B18 7QH, UK

Tel: + 44 (0)785 234 2072;

Fax: + 44 (0)709 2089521

E-mail: a.manna@nhs.net

Received: 11 March 2011 Accepted in revised form: 24 November 2011

Published online:

3 February 2012
Eye (2012) 26, 742-745; doi:10.1038/eye.2011.361; published online 3 February 2012

Keywords: cyclodiode; glaucoma; glaucoma, angle-closure; intraocular pressure; laser coagulation; treatment outcome

\section{Introduction}

Acute symptomatic angle closure (AAC) is a sight-threatening ophthalmic emergency.
A Manna ${ }^{1}$, P Foster ${ }^{2}$, M Papadopoulos ${ }^{2}$

and W Nolan ${ }^{1}$

The aim of treatment in AAC is to lower the intraocular pressure (IOP) and reverse the underlying mechanism. Techniques such as topical and systemic IOP-lowering medication, argon laser peripheral iridoplasty, ${ }^{1}$ and paracentesis $^{2}$ are sometimes employed to break the attack. Laser iridotomy ${ }^{3}$ is the definitive treatment of AAC once the attack is resolved.

In cases where the IOP does not settle in spite of conventional therapy, surgical interventions including lensectomy, ${ }^{4}$ goniosynechialysis, ${ }^{5}$ and trabeculectomy ${ }^{6}$ may be effective. However, lensectomy can be technically challenging with a risk of complications including suprachoroidal haemorrhage and decompression retinopathy. ${ }^{7}$ Trabeculectomy in this setting has a high risk of surgical failure and postoperative complications. ${ }^{6}$ We describe five cases of AAC refractory to conventional treatment where diode laser transscleral cyclophotocoagulation (cyclodiode laser) achieved successful control of IOP and resolution of the acute attack.

\section{Case reports}

Three consultants (PF, MP, WN) from two ophthalmic units kept a record of patients under their care requiring urgent cyclodiode laser for AAC between January 2008 and February 2009. At the end of this period, five patients were identified and a retrospective case note review was undertaken. Demographic information, symptoms, medical and surgical treatment, visual outcomes, and IOP were recorded.

A diode laser equipped with a G-probe (Iris Medical Instruments, Mountain View, CA, USA) was used to deliver transscleral cyclophotocoagulation.

The key features of these cases are summarised in Table 1.

All patients were refractory to conventional treatment (IOP-lowering medication), some of whom also underwent laser iridotomy, paracentesis, and iridoplasty, as detailed in Table 1. 


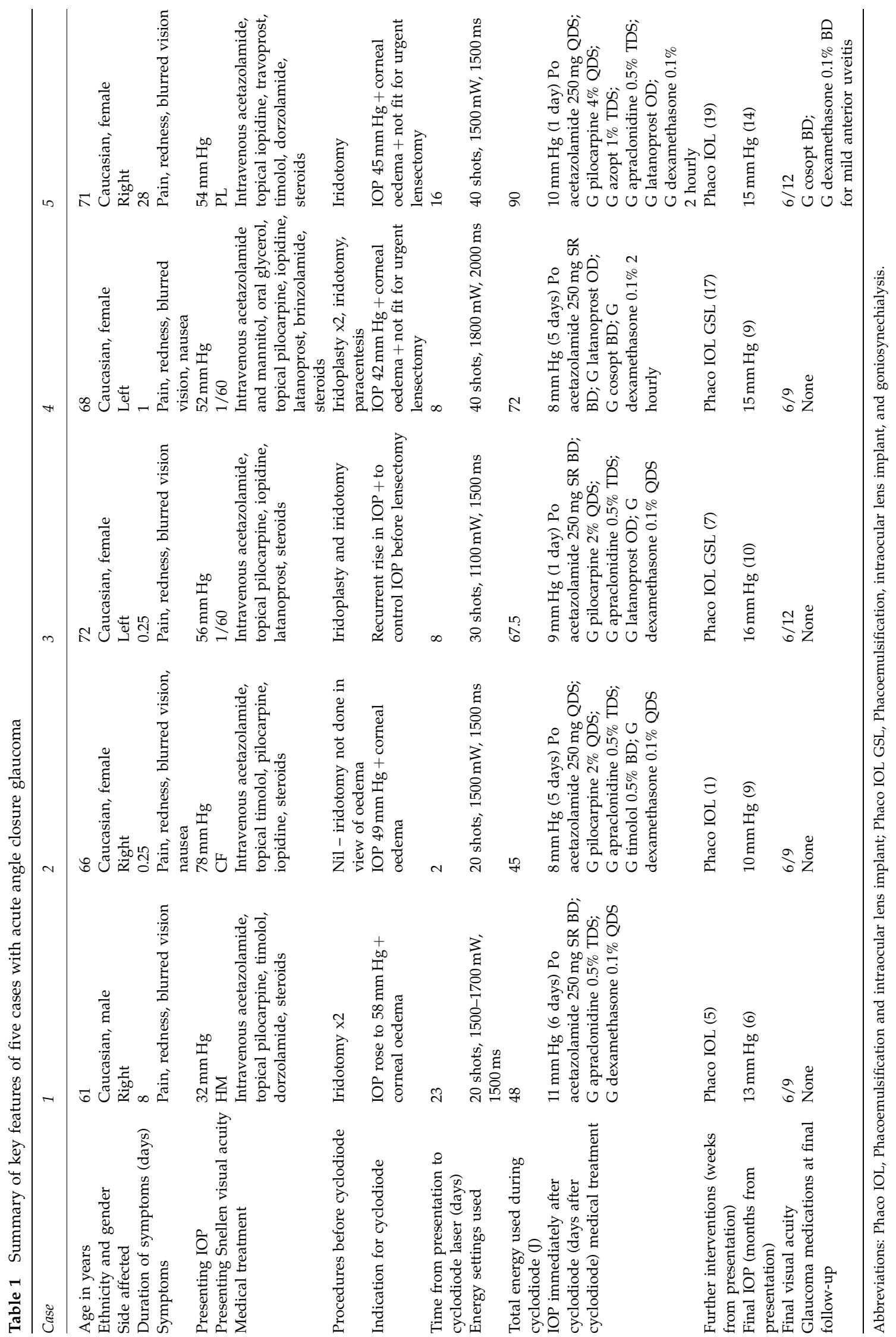


All patients underwent phacoemulsification and lens implant following cyclodiode laser and two also underwent goniosynechialysis.

Follow-up ranged between 6 and 14 months and all patients had satisfactory IOP at their final visit. All patients had better vision at follow-up compared with presentation. One patient (case 5) developed a low-grade chronic anterior uveitis at the last visit (14 months after presentation) and was started on topical steroid drops. No other complications were documented. Only one of the five patients needed IOP-lowering topical treatment at the final visit (case 5).

In all cases, cyclodiode laser allowed cessation of oral acetazolamide before lens extraction.

\section{Discussion}

Cyclodiode laser has been described in the management of medically uncontrolled primary angle closure glaucoma (PACG). ${ }^{8,9}$ This is to our knowledge the first report of using cyclodiode laser for the management of AAC where conventional treatment was unsuccessful. In all cases, the patients regained normal IOP and good visual function.

A limitation of our case series is that the laser settings used varied as different operators had different preferences. This is in concordance with a recent survey that shows variation of laser settings within the United Kingdom. ${ }^{10}$ Furthermore, this case series is too small to be able to judge whether the pressure-lowering effect is related to the total energy used.

Potential complications of cyclodiode laser include temporary conjunctival surface burns, postoperative pain, intraocular haemorrhage, prolonged ocular inflammation, corneal oedema, and hypotony. ${ }^{8,9,11}$ Case reports describe malignant glaucoma, lens subluxation and scleral perforation following cyclodiode laser. In two papers reporting the use of cyclodiode laser for PACG, complications of bullous keratopathy and atonic pupil were recorded..$^{8,9}$ However, the only adverse event in our paper is a mild anterior uveitis that occurred 14 months after presentation. It is possible that the inflammation was related to the higher laser energy used. Because of the time delay from laser treatment and the incidence of inflammation, other factors such as delayed presentation and cataract surgery may have also contributed to the intraocular inflammation.

The alternatives to cyclodiode for AAC are lensectomy and trabeculectomy. Both pose surgical challenges in the presence of AAC and are associated with complications. The complications of lensectomy after abortion of AAC are: intraoperative corneal oedema, posterior capsular rupture, intraoperative bleeding from iris root, postoperative fibrinous $\mathrm{AC}$ reaction, and visually significant posterior capsular opacification. ${ }^{4}$ Lensectomy performed before abortion of AAC is likely to entail greater risks. Trabeculectomy in the presence of medically uncontrolled AAC has a $65.6 \%$ chance of success (IOP $<21 \mathrm{~mm} \mathrm{Hg}$ with or without medication) at a mean follow-up of 22 months. ${ }^{6}$ Complications include shallow anterior chamber, transient IOP elevation, and hyphaema. ${ }^{6}$

In conclusion, we feel that cyclodiode laser is a safe alternative to lensectomy or trabeculectomy in cases of AAC, which are unresponsive to first-line medical and laser therapy. It has the advantages of being quick and easy to perform in an emergency setting and does not entail the more serious risks of an open surgical procedure.

\section{Summary}

\section{What was known before}

- Acute angle closure is a common condition in opthalmological practice all over the UK. Conventional management with pressure-lowering medications and laser iridotomies will usually treat most patients. However, cases refractory to conventional treatment are challenging to manage. Most ophthalmologists would consider emergency trabeculectomy or lensectomy, both of which are suboptimal in an eye with acute angle closure.

\section{What this study adds}

- We propose a better alternative: cyclodiode laser. We demonstrate a good result in five consecutive patients refractory to conventional treatment and who underwent cyclodiode laser. This is a quick and effective treatment that is easily performed in an emergency setting.

\section{Conflict of interest}

The authors declare no conflict of interest.

\section{References}

1 Lai JSM, Tham CCY, Chua JKH, Poon ASY, Lam DSC. Laser peripheral iridoplasty as initial treatment of acute attack of primary angle-closure: a long-term follow-up study. J Glaucoma 2002; 11: 484-487.

2 Lam DSC, Chua JKH, Tham CCY, Lai JSM. Efficacy and safety of immediate anterior chamber paracentesis in the treatment of acute primary angle-closure glaucoma: a pilot study. Ophthalmology 2002; 109: 64-70.

3 Aung T, Ang LP, Chan SP, Chew PT. Acute primary angleclosure: long-term intraocular pressure outcome in Asian eyes. Am J Ophthalmol 2001; 131: 7-12.

4 Lam DSC, Leung DY, Tham CC, Li FC, Kwong YY, Chiu TY et al. Randomized trial of early phacoemulsification $v s$ peripheral iridotomy to prevent intraocular pressure rise after acute primary angle closure. Ophthalmology 2008; 115: 1134-1140. 
5 Teekhasaenee C, Ritch R. Combined phacoemulsification and goniosynechialysis for uncontrolled chronic angleclosure glaucoma after acute angle-closure glaucoma. Ophthalmology 1999; 106: 669-675.

6 Aung T, Tow SLC, Yap E-Y, Chan S-P, Seah SK. Trabeculectomy for acute primary angle closure. Ophthalmology 2000; 107: 1298-1302.

7 Dudley DF, Leen MM, Kinyoun JL, Mills RP. Retinal hemorrhages associated with ocular decompression after glaucoma surgery. Ophthalmic Surg Lasers 1996; 27: $147-150$.

8 Lai JSM, Tham CCY, Chan JCH, Lam DSC. Diode laser transscleral cyclophotocoagulation as primary surgical treatment for medically uncontrolled chronic angle closure glaucoma: long-term clinical outcomes. J Glaucoma 2005; 14: 114-119.

9 Lai JSM, Tham CCY, Chan JCH, Lam DSC. Diode laser transscleral cyclophotocoagulation in the treatment of chronic angle-closure glaucoma: a preliminary study. J Glaucoma 2003; 12: 360-364.

10 Agrawal P, Dulku S, Nolan W, Sung V. The UK national cyclodiode laser survey. Eye (Lond) 2011; 25: 168-173.

11 Pastor SA, Singh K, Lee DA, Juzych MS, Lin SC, Netland PA et al. Cyclophotocoagulation: a report by the American Academy of Ophthalmology. Ophthalmology 2001; 108: 2130-2138. 